\title{
Neoclassical Effects on Solar Plasma Loops
}

\author{
B.L. $\operatorname{Tan}^{1,2} \dagger$ and G.L. Huang ${ }^{1}$ \\ ${ }^{1}$ Purple Mountain Observatory CAS, Nanjing 210008, China \\ email: bltan@pmo.ac.cn \\ ${ }^{2}$ Graduate School of the CAS, Beijing, China
}

\begin{abstract}
Tokamak physics shows that there will be a neoclassical effect in current-carrying plasma loops. We apply the theory to solar coronal loops and hope to find a fast magnetic reconnection mechanism for understanding solar flares and CMEs.
\end{abstract}

Keywords. Sun: coronal mass ejections (CMEs), flares, magnetic fields, MHD

\section{Introduction}

Studies on Tokamak show, in current-carrying plasma loop, the transport coefficient of energy and particles is much greater than that of the loop without current, and will trigger a new kind of MHD instabilities-neoclassical tearing mode (NTM). These phenomena can be called neoclassical effect. It includes: (A) Neoclassical resistance (Wesson 1997), $\eta^{n c}=\eta^{s p} /(1-\sqrt{\epsilon})^{2}, \epsilon=\mathrm{a} / \mathrm{R}, \eta^{s p}$ is classical resistance. (B) Bootstrap current (Bickerton et al. 1971): $\mathrm{J} b=-2 \sqrt{2} \frac{\epsilon^{1 / 2} T}{B_{\theta}} \frac{d n}{d r}$. Since 1988, in large Tokamaks, bootstrap current is observed up to 0.85MA, and the fraction $f_{b}$ is up to $77 \%$ (Wesson 1997).(C) Neoclassical Tearing mode $(N T M)$ :When $f_{b}$ is much enough, NTM will be triggered(Qu and Callen, 1985). Its growth rate is: $\gamma_{N T M}=6.61\left(\tau_{h}\right)^{-1} \Delta^{-\frac{2}{3}}\left(j_{b} \alpha \dot{B}_{\theta}\right)^{\frac{2}{3}} S^{-\frac{1}{3}}$. S is magnetic Reynolds number, $\Delta$ is tearing mode instability factor, $\alpha$ is mode number. As a contrast, the classical tearing mode $(C T M)$ is: $\gamma_{C T M}=0.755\left(\tau_{h}\right)^{-1} \Delta^{\frac{4}{5}}\left(\alpha \dot{B}_{\theta}\right)^{\frac{2}{5}} S^{-\frac{3}{5}}$. For solar plasma, $\mathrm{S} \sim 10^{8}-10^{11}, \gamma_{N T M} \sim 10^{2}-10^{3} \gamma_{C T M}$.

\section{Analysis on solar coronal loops}

Typical coronal loops: Similarly, coronal loop are always current-carrying plasma loops, It is reasonable to suppose that neoclassical effect will play a role in the evolution of such loops. The first \& second column in table 1 is the parameters in typical coronal loops (Zhang,2000) and calculating results. Loop's current is typical value (Zaitsev et al. 1998, Khodachenko et al. 2003). We can find, in typical cool loops $f_{b}<0.3 \%$, and in hot loops $f_{b}<2 \%$. Tokamak experiences show neoclassical effect isn't obvious when $f_{b}<20 \%$, we can't detect any measurable information about it. This shows in typical coronal loops the neoclassical effect is so faint that we may neglect it.

A special solar coronal loop: In fact, the range of coronal loops' parameters is always very large. It is reasonable to suppose that in some special loops the neoclassical effect may become measurable. The third column in table 1 is a M3.6 GOES flare event on 25 August 1999 from 01:32 UT to 01:40 UT in AR8674 (S28E21). (Huang et al. 2003). Figure 1(a) is the image, from it we may estimate the geometrical parameters. Figure 1(b) shows the time profile of GOES 8 X-ray. It reflects the thermal emission feature. The duration of rising phase is about 4 minutes. The bootstrap current is $f_{b}=29.1 \%$, this

$\dagger$ Present address: No.2, West Beijing road, Nanjing 210008, China. 
Table 1. (Loop's parameters and bootstrap current calculating results)

\begin{tabular}{lccc}
\hline Parameters & Typical cool loop & Typical hot loop & Special loop \\
\hline Loop radius $(m)$ & $1.0 \times 10^{7}$ & $5.0 \times 10^{7}$ & $7.0 \times 10^{6}$ \\
Section radius $(m)$ & $1.0 \times 10^{6}$ & $5.0 \times 10^{6}$ & $2.8 \times 10^{6}$ \\
Plasma temperature $(\mathrm{eV})$ & 10 & 200 & 900 \\
Loop magnetic field & 0.1 & 0.1 & 0.1 \\
Plasma mean density & $1.0 \times 10^{16}$ & $2.0 \times 10^{15}$ & $1.3 \times 10^{16}$ \\
Electric current $(A)$ & $4.0 \times 10^{10}$ & $1.0 \times 10^{11}$ & $1.0 \times 10^{11}$ \\
e mean free path $(\mathrm{m})$ & $1.02 \times 10^{4}$ & $2.05 \times 10^{7}$ & $6.4 \times 10^{7}$ \\
Bootstrap current, $j_{b}\left(\mathrm{~A} / \mathrm{m}^{-2}\right)$ & $3.79 \times 10^{-5}$ & $2.02 \times 10^{-5}$ & $1.17 \times 10^{-3}$ \\
Fraction, $f_{b}(\%)$ & 0.299 & 1.59 & 29.1 \\
\hline
\end{tabular}

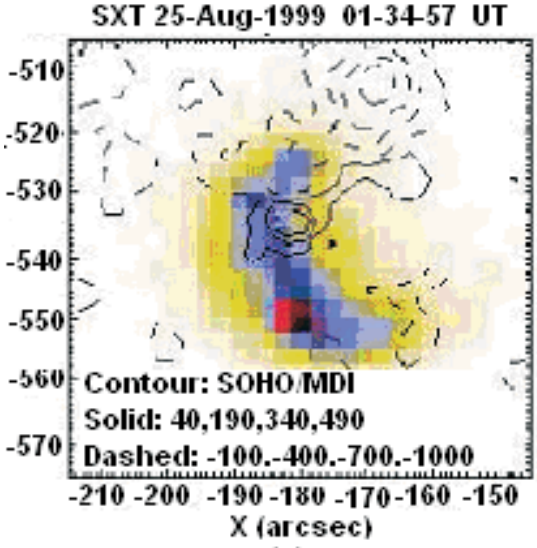

(a)

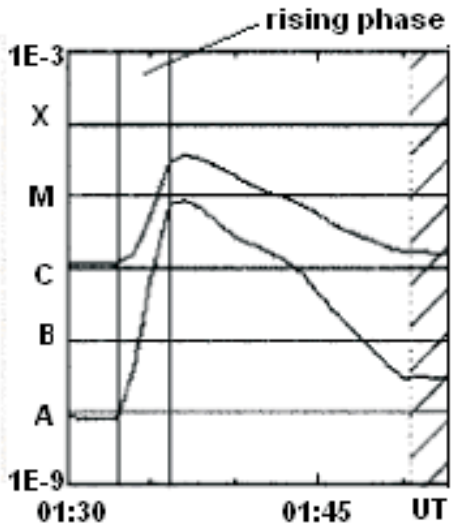

(b)

Figure 1. A special coronal loop and its emission feature

value is higher than that of JET in 1989's experiments. As the neoclassical effect is proportion to $f_{b}$, the neoclassical effect in above loop is very obvious. Similar to tokamak experience, NTM will be triggered. Then we may get: $\gamma_{N T M}=2.18 \times 10^{-3}, t_{N T M}=$ $4.5 \times 10^{2}(s), \gamma_{C T M}=1.38 \times 10^{-6}, t_{C T M}=7.2 \times 10^{5}(s)$. We find that NTM is more consistent with the observations than that of CTM.

\section{Conclusions}

(1) In typical coronal loops $f_{b}<2 \%$, it is too small to consider the neoclassical effect.

(2) For special coronal loops, bootstrap current exists well and truly, $f_{b}$ may be over $29 \%$, the neoclassical effect will play an important role in the loop's evolution. NTM will be triggered and provide a mechanism of fast magnetic reconnection.

\section{References}

Bickerton, R.J., Connor, J.W. \& Taylor, J.B. 1975, Nature 229, 110

Huang, G.L., Wu, H.A., Grechnev, V.V., Sych, R.A. \& Altyntsev, A.T. 2003, Sol.Phys. 213, 341 Khodachenko, M., Haerendel, G. \& Rucker, H.O. 2003, Astron.Astrophys. 401, 721 Qu, W.X. \& Callen, J.D. 1985, UWPR-85-5, University of Wisconsin, Wisconsin. Wesson, J. 1997, Tokamak, Claarendon Press, Oxford.

Zaitsev, V.V., Stepanov, A.V., Urpo, S. \& Pohjolainen, S. 1998, Astron.Astrophys. 337, 887 Zhang, Z.D. 2000, Coronal Physics(in Chinese), Science Press, Beijing. 